\title{
MUNICIPAL SOLID WASTE GENERATION MODELLING BASED ON FUZZY LOGIC
}

\author{
NIKOLAOS V. KARADIMAS \\ VASSILI LOUMOS \\ National Technical University of Athens \\ Multimedia Technology Laboratory \\ 9 Heroon Polytechneiou \\ Zografou Campus, 15780 Athens, Greece \\ E-mail: NKaradim@central.ntua.gr \\ E-mail: loumos@cs.ntua.gr
}

\author{
ALESSANDRA ORSONI \\ Kingston University \\ School of Business Information Management \\ Kingston Hill, Surrey KT2 7LB \\ United Kingdom \\ E-mail: A.Orsoni@kingston.ac.uk
}

\section{KEYWORDS}

Waste Model, Fuzzy Logic, Spatial Geodatabase.

\begin{abstract}
The present paper introduces an innovative model for the estimation of urban solid waste productivity using an intelligent system based on fuzzy logic. The model retrieves the required information from a spatial Geodatabase, integrated in a GIS environment.

The model takes into consideration several parameters of waste production, such as population density, maximum building density, commercial traffic, area and type of shops, road network and its relative information (e.g. road width, dead-end streets, etc) linked with the allocation of waste bins. Additionally, ground-based analysis has been applied for the estimation of the interrelations between the aforementioned factors and the variations in waste production between residential and commercial areas.

Therefore, the proposed model follows a unified and correlated categorization approach for all commercial and industrial activities in the area of study using a weighting system for all of the considered factors. The first results from testing the system using different regions, show the effectiveness of the system in the estimation process of the optimal number of waste bins in each region.
\end{abstract}

\section{INTRODUCTION}

Urbanization is one of the most evident global changes worldwide. The rapid and constant growth of urban population has led to a dramatic increase in urban solid waste production, with a crucial socio-economic and environmental impact. However, the growing concern for environmental issues and the need for sustainable development have moved the management of solid waste to the forefront of the public agenda. Legislation and regulations have been introduced in local and national levels to guide waste management, and techniques for appropriate waste treatment and disposal have been developed. Moreover, strategies for sustainable waste management have emphasised the requirement to minimise waste production, increase waste recovery and reduce the use of landfill sites.

Nowadays, there is a general agreement on the best practices for sustainable management of urban solid waste, but only isolated efforts have been made so far in this domain, which were adapted to specific regulations and needs of each national or regional authority (Leao et al, 2001).

However, sustainable waste management seems far from being attained. Reduction of waste production is still a hope more than an achievement in most countries. The net waste production increases as population grows, and per capita generation of waste is also increasing, particularly in developing countries. Disposal in landfills is still the most usual solution.

Waste management issues should be confronted in a more generalized manner, which means that new strategies need to be designed for considering diverse and variable urban models. This fact demonstrates the necessity of developing integrated, computerized systems for obtaining more generalized, optimal solutions for the management of urban solid waste (Karadimas et al, 2004).

The current work describes an innovative model that can lead to the prediction and computation of the municipal solid waste generation and consequently to the calculation of the optimal number of waste bin as well as their position in a pre-defined area.

The paper is structured in the following sections: Section 2, presents a theoretical overview of the related literature. In Section 3, the waste management problem in the selected case study area is presented and Section 4 describes the solid waste generation factors. In Section 5 an overview of fuzzy sets and fuzzy logic is introduced. Section 6 illustrates the fuzzy inference system while Section 7 is devoted to conclusions and future prospects.

\section{OVERVIEW}

The estimation and the prediction of municipal solid waste generation plays an important role in solid waste management. The population growth and migration, underlying economic development, household size, 
employment changes, as well as the impact of waste recycling are factors that influence solid waste generation interactively. The development of a reliable model for calculating and subsequently predicting the aggregate impact of economic trends, population changes, and recycling impact on solid waste generation would be a useful advance in the practice of solid waste management.

The issue of determining a suitable procedure of analysis for municipal solid waste (MSW) quantities has been investigated in many research approaches. Certain aspects of MSW management and policies are found in the literature based on the correlation among socioeconomic data and solid waste composition and quantities. The main objective in these cases is to simulate the multivariate problem of MSW in a mathematical form by using deterministic models (mostly regression models) and trend analysis (Karadimas et al, 2005).

DeGeare and Ongerth (1971), performed a multiple stepwise regression analysis in an effort to predict from the existing data sets the weekly quantities of collected waste. On the contrary, Rhyner (1992) analyzed the monthly quantities of solid waste (urban, industrial, etc) generated in Wisconsin by using mean values and standard deviations between 1985-1989. Matsuto and Tanaka (1993) have normalized values of waste amounts and subsequently have estimated moving averages. The advantage of this analysis was that the moving average curve showed seasonal variations, in most cases, due to the residents' behavior. Grossman et al. (1974) includes the effects of population, income level, and the dwelling unit size in a linear regression model. Niessen and Alsobrook (1972) conducted similar estimates by providing some other extensive variables characterizing waste generation. But dynamic properties in the process of solid waste generation cannot be fully characterized in those model formulations.

To implement those traditional statistical estimation methods, however, it would require collecting thorough socioeconomic and environmental information before the estimation analysis can be performed. In many cases, municipalities might not have a sufficient budget and management capacity to maintain a complete database of solid waste quantity and quality in support of such needs on a long-term basis.

Most traditional statistical estimation models, such as the geometry average method, saturation curve method, least-squares regression method, and the curve extension method, are designed based on the configuration of semi-empirical mathematical models. The structure of these models is simply an expression of cause-effect or an illustration of trend extension in order to verify the inherent systematic features that are recognized as related to the observed database.

In addition to short-term forecasting, long-term forecasting could also be very useful to waste management authorities or companies in terms of selecting appropriate technologies or scheduling landfill sites. However, when estimating long-term MSW production rates, one should be aware of significant changes either in the attitudes of consumers/waste generators or in national or local decrees and regulations regarding waste minimization and recycling. Therefore, estimations should be based on techniques that identify trends and seasonality, and give recent data higher consideration.

In light of the evolution of structured or semi-structured estimation techniques, the synergy of fuzzy logic modelling is viewed as a promising approach for handling estimation issues under uncertainty.

\section{CASE STUDY}

In this research work, a small part of Attica's prefecture (a suburb of Athens) was chosen as the case study area. The municipality of Athens is empirically divided into about 145 solid waste collecting programs. Figure 1 illustrates one of these collecting programs, in which each one includes approximately 100 waste bins. The waste disposal truck, which is responsible for the collection of the solid waste in that particular area, must visit all the bins in order to complete its collection program.

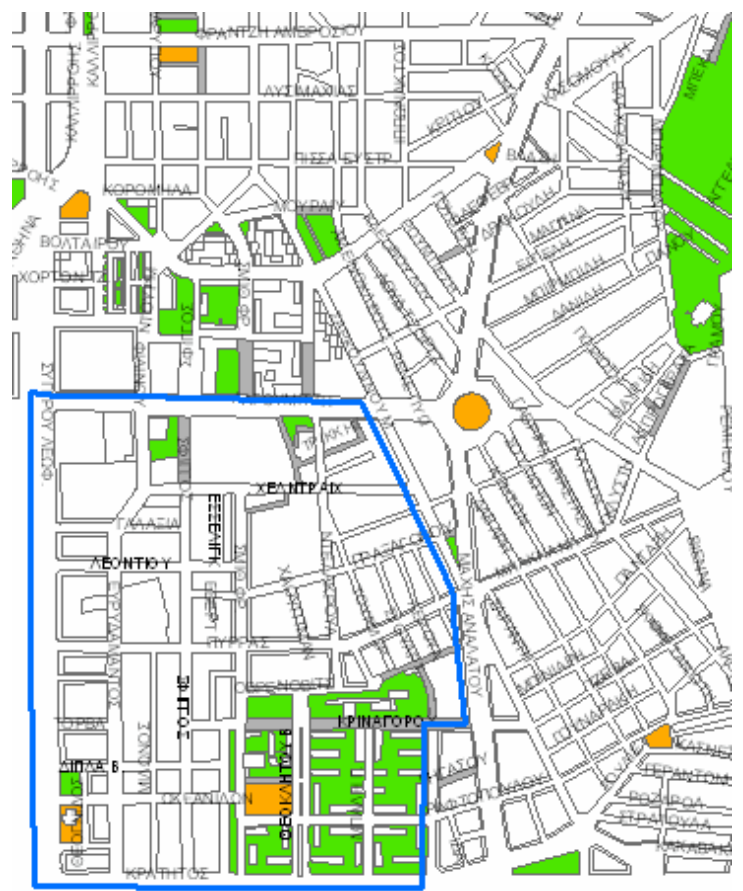

Figure 1: The area of Athens used in our experiments

The examined area is about $0.45 \mathrm{~km}^{2}$, with a population of more than 8,500 citizens and a production of about 3,800 tones of solid urban waste per year. The data concerning the area under examination was obtained from the pertinent Agency of the Municipality of Athens. The data includes maps of the examined area with the correspondent annotation (address and numbering labels of streets), the building blocks and the locations of the existing waste bins. 
The waste bin locations were initially derived from a pilot program that the municipality of Athens was using for the allocation of their trucks. For that purpose Global Positioning System (GPS) and General Packet Radio Service (GPRS) technologies have been used and the information received was recorded into a spatiotemporal database.

To estimate the daily waste generation in association with the population and commercial distribution in an area, the number of citizens per building block and the factors which affect the daily waste production per citizen ( $\mathrm{kgr} /$ citizen) should be provided as well as the number of the commercial activities and their daily waste production per activity.

The average daily waste production number, that has been proposed by the Municipality was $1.2 \mathrm{kgr} / \mathrm{citizen}$, has been considered as unrealistic due to the unreliable method of the calculation performed by the Municipality.

The calculation method that has been used was the following: When a truck approaches the landfill, it undergoes weighing and at the end of the day, the sum of all loadings provides the overall daily waste production for the total of residents. The daily waste production per citizen was derived dividing the overall waste loading by the number of citizens in the area under examination. Nevertheless, commercial units (such as Super Markets and others) produce a significant quantity of waste relative to their type and the fact is that the empirical model used by the municipality, does not specify the quantity of the waste due to these commercial factors. This parameter plays an important role because of its impact on the remaining factors for the computation of the overall cost.

For example, an area with high commercial traffic, but at the same time, with a small number of citizens, probably generates more solid waste in a daily basis than if it was a strictly residential area.

Such constraints should be taken into account in order to design a successful waste collection process. To achieve optimum benefits from waste collection services, an appropriate positioning of the waste bins on the map is required.

The waste bins used by the Municipality of Athens have a total capacity of $1.10 \mathrm{~m}^{3}$ with $90 \%$ of fullness. According to that, and the fact that the density of urban solid waste is about $130 \mathrm{kgr} / \mathrm{m}^{3}$, the quantity of waste in each bin should be approximately 120-130 kgr $\approx 125 \mathrm{kgr}$. These bins should also be set in a way that citizens are not obliged to cover a long distance from their homes to dispose the waste. Simultaneously, the number of bins needs to be proportional to the number of citizens, so that no bin remains empty throughout the day, as this might increase the collection cost.

Furthermore, the solid waste is collected every day by the Municipal services. For all the aforementioned reasons, for every group of regions, the corresponding daily waste loading should be calculated.

Of course, it is very difficult to assign a set of coefficients, which directly affect the daily waste generation and can precisely estimate their weight for the total solid waste of a particular area, because there are some uncertain parameters such as: the real-estate commercial value and the maximum building density factor of the particular segment area. Moreover, in that phase, it is very hard to collect all the electricity bills from every property in the area. However, electricity bills could be an important factor for waste estimation, because in many cases, there is a relation between waste generation and electricity consumption. Furthermore, the commercial traffic in a particular area is another factor that is not precisely estimated, as well as the area that is used for each commercial activity.

On the contrary, the value of the daily waste production per citizen is calculated as a range between 0.8 and $1.2 \mathrm{kgr} /$ citizen, depending on the area in which each citizen lives and it is estimated on ground-based statistical analysis, and this set of values concerns strictly residential areas (without any commercial or industrial activity).

Having in mind all the aforementioned factors, this makes fuzzy logic a more obvious approach to this problem. Fuzzy logic was introduced by Zadeh (1965) to describe and to deal with such uncertainties. In this work, the power of fuzzy logic and fuzzy inference systems were utilized for the estimation of the total urban solid waste generation.

All of the coefficients are represented by linguistic variables. Then these coefficients are weighted to represent the importance and the contribution of each factor. Thus, the proposed system encodes the expertise of the environmentalists in a fuzzy inference system in order to estimate the total waste. The system can be easily used by MSW planners due to waste bins calculation and their allocation in a pre-defined area.

The system is flexible and can be adapted for new information on the pre-defined area that may be incorporated into a knowledge base.

This will allow the user's interaction with the computer in order to understand better the initial waste bins calculation process. Several factors were considered in the solid waste generation process. These factors cover commercial traffic, electricity bills, commercial area (in $\mathrm{m}^{2}$ ), maximum building density factor, real-estate objective values and others.

\section{SOLID WASTE GENERATION FACTORS}

In assessing the total solid waste generation in an area under study, many factors should be considered. These factors may be presented in many ways; however the most useful way is the one that can easily be understood by the community. The following factors are usually considered in the solid waste generation process:

- real-estate commercial values 
- maximum building density factor

- electricity bills

- commercial traffic

- land use

- area in square meters $\left(\mathrm{m}^{2}\right)$

In addition to the factors previously mentioned, other factors may be considered based on local conditions and circumstances. In this work, the factors used for the analysis of the total solid waste generation are grouped into two main categories, including the estimation of the daily residential waste generation as well as the commercial - industrial generation of solid waste in the area under study.

The residential waste coefficients include real-estate commercial values (RECV), maximum building density factors (MBDF), size of area in square meters (SA) and electricity bills (EB). On the other hand, the commercial coefficient includes factors related to commercial traffic (CT), land use (LU), size of area in square meters (SA) and the electricity bills (EB) of each commercial property.

The contribution and severity of the aforementioned various factors in the solid waste estimation may be different. For example, the same commercial activities, in different areas, with different commercial traffic factors may produce completely different amounts of solid waste.

The impact severity of each parameter was determined as Big, Medium and Small. Among the many aspects to be considered for the calculation of the total solid waste generation, it is important to consider most of them with objective weighting. The degrees of weightiness importance for a specific factor may be different. A scoring system is commonly used to express this variability. In the present study, these factors were given weights based on a scoring system ranging from 0 to 1 . These weights were given based on a consensus of professionals with expertise in solid waste estimating and on data from the technical literature.

\section{FUZZY SETS AND FUZZY LOGIC}

Uncertainty is a major concept in our daily life. Fuzzy sets and fuzzy logic provide an approach to deal with this concept. Fuzzy logic modeling supports an easier transition between human thinking and computer-based analysis in complex decision processes involving the assessment of uncertain information.

In recent years, fuzzy logic has been successfully applied in a variety of disciplines including computer vision, weather prediction, image processing, nuclear reactor control, control of biomedical processes, automatic tuning and many other fields of research.

Fuzzy sets are sets with imprecise boundaries. A fuzzy set provides a mechanism to express the degree of membership rather than accepting or denying the membership. The wide use and popularity of fuzzy set is related to its ability to tolerate imprecise and linguistic data. It assigns each element in the universe of discourse a value representing its grade of membership in the fuzzy set. This number represents the certainty or belief that this individual is compatible with the concept represented by the fuzzy set. A membership function (MF) is a curve that maps each element in the input space into a membership value called the degree of membership.

The only restriction on the MF is that it must vary between 0 and 1 . The function itself may take any shape that is defined and specified by the designer to suit the nature of the problem from the point of view of simplicity, convenience, speed and efficiency.

One of the most common classes of MFs is the trapezoidal MF. The trapezoidal membership function has a flat top and really is just a truncated triangle curve. A trapezoidal MF can be specified by a collection of four points $\{\mathrm{a}, \mathrm{b}, \mathrm{c}, \mathrm{d}\}$. It is expressed as:

$$
\mu(x)=\left\{\begin{array}{ll}
0, & x \leq a, \quad d \leq x \\
\frac{x-a}{b-a}, & a \leq x \leq b \\
1, & b \leq x \leq c \\
\frac{d-x}{d-c}, & c \leq x \leq d
\end{array}\right\}
$$

where $(\mathrm{a}<\mathrm{b}<\mathrm{c}<\mathrm{d})$ are the $\mathrm{X}$ coordinates of the four corners of the underlying MF. Fuzzy sets representation conforms to the objectives appearing in our daily linguistic usage such as sets of \{Low-Medium-High\}, \{Small-Medium-Big\}, etc. These expressions are called linguistic values and the universe field of discourse on which these values are defined on is called a linguistic variable. The basic operations on a classical set are union, intersection and complement. Corresponding to those basic operations, Zadeh defined similar operations on fuzzy sets (Zadeh, 1965). Fuzzy IF-THEN rules form the rule base in a fuzzy inference system and they provide means to encode conditional propositions. A single IF-THEN rule takes the form:

IF commercial traffic is high and area is low THEN... All parts of the antecedent are evaluated simultaneously and resolved to a single number using the logical operators (AND, OR, NOT).

Fuzzy reasoning, known also as approximate reasoning, is the process of deriving conclusions from a set of IFTHEN fuzzy rules using an inference procedure. By fuzzy reasoning, the truth of the consequent is inferred from the degree of truth of the antecedent.

The concept of fuzzy set theory, IF-THEN rules, and fuzzy reasoning together constitute a computing framework usually called fuzzy inference system (FIS).

The structure of a fuzzy inference system consists of three major parts: a rule base that holds the fuzzy IFTHEN rules used in the inference process, a database that contains the membership functions that characterize the fuzzy sets, and a reasoning mechanism that performs the inference procedure and derives conclusions depending on a set of rules and facts. The fuzzy 
inference process consists of five steps including fuzzification, application of the fuzzy operators, fuzzy implication, fuzzy aggregation, and defuzzification. The last step uses a defuzzification method to produce a single crisp number for each output variable.

There are three widely used types of fuzzy inference systems: Mamdani, Sugeno, and Tsukamoto. The basic difference between various models lies in the representation of the consequents of their fuzzy rules. Accordingly, the aggregation and defuzzification procedures of the three models are different. In this work, the Mamdani model will be utilized in developing the system, as this is the most suitable model for encoding experts opinion in which consequences are expressed as linguistic variables, which is the case in this work.

The Mamdani model was proposed by Mamdani in 1975 to control a steam engine and boiler combination. It was among the very first control systems built based on the theory of fuzzy logic (Mamdani and Assilian, 1975). This model is characterized by representing the consequents of its rules using fuzzy sets. The aggregation of the outputs of all rules yields a single fuzzy set (output). A defuzzification process is then performed to extract a crisp value from the output fuzzy set. For example, using the centroid defuzzification method, the crisp output of the Mamdani fuzzy inference system is given by:

$$
D e f_{y}=\frac{\int_{Y} \mu_{\mathrm{B}^{\prime}}(y) y d(y)}{\int_{Y} \mu_{\mathrm{B}^{\prime}}(y) d(y)}
$$

\section{MSW ESTIMATION USING FUZZY INFERENCE}

The proposed system utilizes the power of fuzzy sets in describing uncertainties in the different factors involved in solid waste estimation. Each factor is represented by a linguistic variable defined over a universe of discourse that represents all permissible values. The expertise of an environmentalist is coded into a fuzzy rule base, which drives the inference system as shown in Figure 2.

The contribution and importance of the various factors in the solid waste calculation may be different. A scoring system is commonly used to express this variability.

The total solid waste generation is expressed as a weighted sum of all factors. These weights are given judgmentally based on a consensus of those professionals with expertise in solid waste generation and on data from the technical literature.

Linguistic values are used for the residential waste coefficient as well as for the commercial coefficient. The rule base consists of rules that describe the various contributions of these factors on the total solid waste generation in an area under study. The final crisp output is given after defuzzification of the aggregated outputs, for both residential and commercial fuzzy inference, using the centroid method as described in Equation (2).

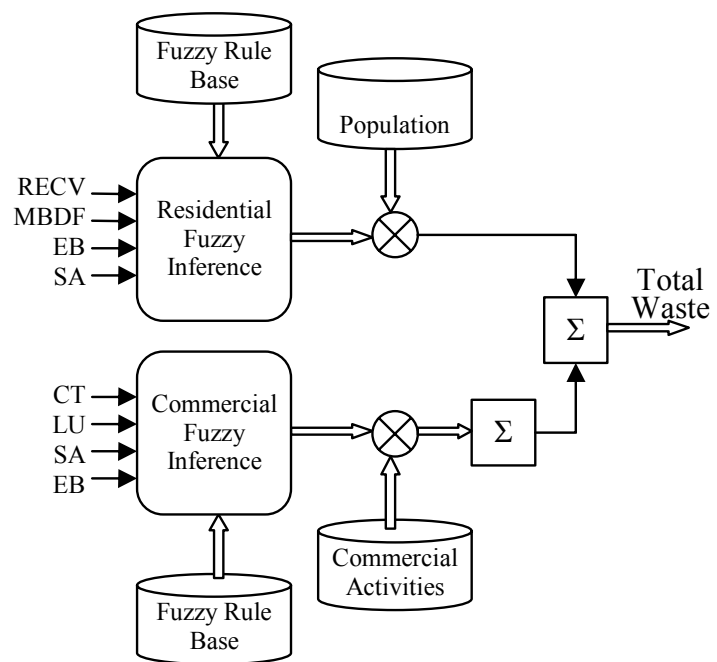

Figure 2: Solid waste estimation using fuzzy inference system

After the defuzzification of each group of the coefficients the following equation (3) is used to calculate the final solid waste generation. The following equation could accurately calculate the total solid waste generation on a given area, based on the population and their waste generation coefficient of the pre-defined region as well as the number and waste generation of the industrial and commercial activities involved.

$$
\text { TotalWaste }=\sum_{i=1}^{m}\left(a_{i} x_{i}+\sum_{j=1}^{n} b_{i j} y_{i j}\right)
$$

where $a_{i}$ is the population of the examined area $i, x_{i}$ is the value of the daily waste production per citizen in the area $i$, produced by the defuzzification method. Also, $b_{i j}$ is the total area (in $\mathrm{m}^{2}$ ) of every commercial activity $j$ in the particular segment area $i$, and $y_{i j}$ is the coefficient related to waste generation of the commercial activity $j$ in the area $i$ produced by the defuzzification method. Furthermore, $m$ is the distinct set of areas, used for the calculation of the total solid waste generation, while $n$ is the total number of commercial activities in each predefined area.

The estimates ( $x_{i}$ and $y_{i j}$ coefficients) can be used to construct the prediction equation waste production and to generate predicted scores on a variable for further analysis. Thus, the resulting waste production equation can be used to estimate the waste production rates in other areas, in order to improve waste management and planning.

\section{DISCUSSION AND FUTURE WORK}

This work focuses on the design and the implementation of an innovative model for accurate estimation of urban solid waste. The model should be practical, taking into account the resources available and should explain clearly and directly the analysis and the results in an easily understandable format. 
In this research work, an intelligent system approach for the calculation of the urban solid waste was utilized. The system uses a fuzzy inference system that can encode the expertise of an environmentalist and it can adapt by adjusting the knowledge base. Several factors that affect the waste generation process were considered including commercial traffic, land use, real-estate commercial values, maximum building density factors and others. A scoring system should be utilized to represent the importance of these factors. The proposed system could rank each output using a scale that ranges all the aforementioned factors.

Experimental results reveal the effectiveness of the system in terms of its ranking capabilities. The proposed system can be used as a tool by planners and decision makers in the process to estimate precisely the solid waste generation in the area under study. That is, because fuzzy reasoning represents better the uncertainty introduced in the calculation of the above coefficients. Thus, the commercial traffic and other related parameters in an area, which are in turn related to waste productivity, are better expressed with a range of values rather than with a single value.

Although the case study covers an area of about $0,45 \mathrm{~km}^{2}, 8500$ citizens and over 100 building blocks, to ensure the reliability of the derived results, the proposed model should be tested in an even more extended area.

\section{REFERENCES}

DeGeare, T.V. and Ongerth, J.E., 1971. Empirical analysis of commercial solid waste generation. J. Sanitary Engrg. Div., 97(SA6), 843-850.

Grossman, D., Hudson, J.F. and Mark, D.H., 1974. Waste generation methods for solid waste collection. J. Environ Eng. ASCE 6, 1219-1230.

Karadimas, N.V., Loumos, V. and Mavrantza O., 2004. Quality of Service Ensuring in Urban Solid Waste Management, in Proc. of the $2^{\text {nd }}$ International IEEE Conference on Intelligent Systems, Varna, Bulgaria, 288292.

Karadimas, N.V., Mavrantza O. and Loumos V., 2005. System dynamics modelling for accurate estimation of municipal solid waste generation. Waste Management [under review process]

Mamdani, E.H., Assilian, S., 1975. An experiment in linguistic synthesis with a fuzzy logic controller International Journal of Man-Machine Studies, 1-13.

Matsuto, T. and Tanaka, N., 1993. Data analysis of daily collection tonnage of residential solid waste in Japan. Waste Management and Research, 11, 333-343.

Niessen, Niessen, W.R., Alsobrook, A.F., 1972. Municipal and industrial refuse: composition and rates. In: Proceedings of National Waste Processing Conference, USA, pp. 112-117.

Rhyner, C.R., 1992. Monthly variations in solid waste generation. Waste Management and Research, 10, 67-71.

Zadeh, L.A., 1965. Fuzzy sets. Information and Control 8, 338-353.

\section{AUTHOR BIOGRAPHIES}

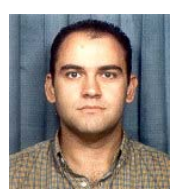

NIKOLAOS V. KARADIMAS was born in Athens, Greece and he graduated from Patra T.E.I. in 1995 with a Bachelor's degree in Electrical Engineering. He then went to receive a Bachelor's degree with Honors in Electronic Engineering and a Masters degree in Computer Science from Glasgow Caledonian University, Scotland in 1997 and 1998, respectively. He also received a Masters degree in Distributed and Multimedia Information Systems from Heriot-Watt University, Scotland in 1999. Since 2002 he is a Ph.D candidate in National Technical University of Athens. Furthermore, since 2001 he is teaching Informatics in Hellenic Army Academy and New York College, since 2003 he is teaching in T.E.I. of Chalkida and since 2005 he is teaching in Technical NCO Academy, as well. He is a member of the Greek Chamber of Engineers, member of IEEE and member of IEE. His research interests are in the fields of Databases, Optimization Techniques, Geographical Information Systems, Information Retrieval, Decision Support Systems and Multimedia.

ALESSANDRA ORSONI is currently a senior lecturer in the School of Business Information Management at Kingston University (Kingston, UK). She received both her MS in Mechanical Engineering and her ScD in Engineering Systems Design and Innovation from the Massachusetts Institute of Technology (Cambridge, MA). Prior to joining Kingston University she was a research associate in the Department of Materials Science and Metallurgy at the University of Cambridge (Cambridge, UK) and worked as an assistant professor in the Department of Production Engineering at the University of Genova (Genova, Italy). Her research interests include modelling, simulation, and AI techniques applied to the design and management of complex industrial systems. 